\title{
25 Research Soure \\ Chest CT Could Be Used to Diagnose 2019 Novel Coronavirus Pneumonia Clinically in Hubei Province
}

\section{Xin Wang}

Jinan Central Hospital Affiliated to Shandong University

Peng Wen

Shandong University

Zhi-Gang Sun ( $\nabla$ sunszg@126.com )

Jinan Central Hospital Affiliated to Shandong University https://orcid.org/0000-0003-1941-250X

Chun-Yan Xing

Jinan Central Hospital Affiliated to Shandong University

Yun Li

Jinan Central Hospital Affiliated to Shandong University

\section{Case report}

Keywords: coronavirus pneumonia, COVID-19, CT, nucleic acid test

Posted Date: March 13th, 2020

DOI: https://doi.org/10.21203/rs.3.rs-17422/v1

License: (c) (i) This work is licensed under a Creative Commons Attribution 4.0 International License.

Read Full License 


\section{Abstract}

Background: In December 2019, novel coronavirus pneumonia-19 (COVID-19) was discovered in the viral pneumonia cases that occurred in Wuhan, Hubei Province, China; and then quickly spread inside and outside of Wuhan and even other countries. This report describes the clinical course of two patients who had COVID-19.

Case presentation: The first case was a typical COVID-19 case. A 66-year-old female presented to our hospital with a 3-day history of fever with coughing, white sputum, runny nose and dizziness. She had contacted with a COVID-19 patient, her daughter-in-law who was diagnosed with COVID-19 two days before. Chest CT showed typical COVID-19 CT imaging. She was diagnosed with COVID-19 by positive nucleic acid test. The second case was a 50-year-old male with a 2-day history of fever and dry coughing. He denied having been to Wuhan. Chest CT also showed typical COVID-19 CT imaging. He accepted COVID-19 nucleic acid test using reverse-transcription polymerase chain reaction of his throat swab sampling 7 times and the test results remained controversial. Eventually, he was diagnosed with COVID19 after 5 days.

Conclusions: Chest CT examination has high sensitivity for diagnosis of COVID-19 clinically, particularly when nucleic acid test is negative. Chest CT should be considered for the COVID-19 screening, comprehensive evaluation and following-up and patients could benefit from effective treatment in time.

\section{Introduction}

In December, 2019, a succession of pneumonia cases, which were later proven to be caused by a novel coronavirus (named as COVID-19 by the World Health Organization (WHO) on 12 January 2020) emerged in Wuhan City, Hubei Province, China. In the following days, the COVID-19 spread inside and outside of Hubei Province quickly and even other countries. A global health emergency was declared by WHO on January 30, 2020[1, 2]. As of March 8th, 2020, a total of 103241COVID-19 cases had been reported in at least 51 countries. Among of them, 80859 cases came from China and 22382 cases came from the other countries. And 3601 patients died of COVID-19 unfortunately. Chest CT is used for diagnosis of COVID-19, as an important complement to the reverse-transcription polymerase chain reaction (RT-PCR) tests. Multiple, patchy, sub-segmental or segmental ground glass density shadows in both lungs are typical COVID-19CT imaging manifestations [3, 4]. Here we report two cases. We hypothesize that chest CT examination is an effective method to diagnose COVID-19 clinically and patients may benefit from effective treatment in time.

\section{Case Report}

Case 1: A 66-year-old female presented to our hospital with a 3-day history of fever with coughing, white sputum, runny nose and dizziness. Two days before, her daughter-in-law was diagnosed with COVID-19. Her body temperature was elevated to $38.6^{\circ} \mathrm{C}$. She was examined for complete blood count, C-reactive 
protein and chest CT . The white cell count was $3.88 \times 109 / \mathrm{L}$ (reducing, range $4-11 \times 109 / \mathrm{L}$ ), with lowered lymphocytes at $1.24 \times 109 / \mathrm{L}$ and normal neutrophils. The C-reactive protein was $10 \mathrm{mg} / \mathrm{L}$ (slightly elevated, normal $<3 \mathrm{mg} / \mathrm{L}$ ). Chest CT showed multiple peripheral solid and ground-glass opacities in both lungs (Figure 1). She was diagnosed with COVID-19 by positive COVID-19. nucleic acid test from her throat and sputum swab sampling using RT-PCR.

Case 2: A 50-year-old male presented to our hospital with a 2-day history of fever and dry coughing. $\mathrm{He}$ denied having been to Wuhan. His body temperature was elevated to $38.5^{\circ} \mathrm{C}$. The examination of complete blood count was normal and C-reactive protein was slightly elevated $(6.39 \mathrm{mg} / \mathrm{L}$, normal $<3$ $\mathrm{mg} / \mathrm{L}$ ). The chest CT result showed multiple peripheral ground-glass image on bilateral lungs (Figure 2). He accepted COVID-19 nucleic acid test using RT-PCR of his throat swab sampling 7 times and the test results remained controversial. The results from our hospital showed negative, positive, negative and positive and the results from Jinan Center for Disease Control and Prevention (CDC) showed negative, negative and positive. Eventually, he was diagnosed with COVID-19 pneumonia after 5 days.

\section{Discussion}

The epidemiological history is very important to diagnosis COVID-19, especially on early age. Guan et al. reported 1099 patients with laboratory-confirmed COVID-19 from 552 hospitals in 30 provinces, autonomous regions, and municipalities in China through January 29, 2020. They found $43.9 \%$ patients were residents of Wuhan. Among the cases who lived outside Wuhan, $72.3 \%$ had contact with residents of Wuhan [5]. Fever, dry cough and fatigue are the most common presenting clinical symptoms of the COVID-19 infected cases. About $20 \%$ of patients are severe, and the mortality is nearly $3 \%[6,7]$.

Chest CT is a very important method to diagnose COVID-19. Typical CT imaging manifestation include: 1) quantity (often more lesions); 2) dominant distribution (mainly subpleural); 3 ) density (mostly uneven, a paving stones-like change mixed with ground glass density and interlobular septal thickening, etc.); 4) shape (large block, patchy, lumpy, nodular, honeycomblike or grid-like, cord-like, etc. Among of them, multiple, patchy, segmental or sub-segmental ground glass density shadows in bilateral lungs are the most common COVID-19 CT images. Some accompany by fine-grid or small honeycomb-like interlobular septa thickening. The high-resolution computed tomography (HRCT) shows the slightly high-density and ground-glass change with fuzzy edge in the fine-grid or small honeycomb-like thickening of interlobular septa. The thinner the chest CT scan layers, the clearer the above imaging manifestations are displayed [3]. Accurate RNA detection of COVID-19 is a diagnostic method close to the gold standard and could be strongly recommended. The first case was a typical COVID-19 case and diagnosed easily on the basis of typical epidemiologic characteristics, typical clinical manifestations, typical chest images, and nucleic acid test.

Recently, the detection of COVID-19 nucleic acid used by RT-PCR has been found to have some shortcomings, as following:1) the nucleic acid detection technology develops immaturely; 2) patient viral load is low; 3 ) different manufacturers may lead to different detection rate; or 4) clinical sampling is 
improper. Fang et al.reported 51 COVID-19 cases, and they found that the diagnosis sensitivity using chest CT image was greater than that using nucleic acid test ( $98 \%$ vs $71 \%, p<.001)[8]$.Tao et al summarized 1014 COVID-19 patients, and their study showed $59 \%(601 / 1014)$ of the cases had positive RT-PCR results, and $88 \%$ (888/1014) had positive chest CT results. The sensitivity of CT in suggesting COVID-19 was $97 \%$ based on positive RT-PCR results [9]. In our study, the second case received COVID-19 nucleic acid test 7 times and the test results remained controversial. He was diagnosed with COVID-19 after 5 days, although his COVID-19 CT imaging manifestation was typical. On February 12, 2020, COVID19 was firstly diagnosed clinically using chest CT in Hubei province according to "diagnosis and treatment of novel coronavirus pneumonia in China (The Fifth Edition, passed on February 5, 2020)". A total of 13332 new cases in Hubei province were clinically confirmed using chest CT without nucleic acid test on one day and received timely treatment (Figure 3).

\section{Conclusion}

In conclusion, our study shows that chest CT examination has high sensitivity for diagnosis of COVID-19 clinically, particularly when nucleic acid test is negative. Chest CT should be considered for the COVID-19 screening, comprehensive evaluation and following-up and patients could benefit from effective treatment in time.

\section{Declarations}

\section{Acknowledgements}

We are deeply saddened by the passing of Dr. Li Wenliang. We all need to celebrate the work that he did on COVID-19.

\section{Funding}

No funding was supported in the study.

\section{Authors' contributions}

WX wrote the case report and collected the data. LY contributed data, provided clinical information, and helped revise the case report. WP provided the radiological images, radiological expertise, and helped revise the case report. CP provided the radiological images and radiological expertise and helped revise the case report. S-ZG and X-CY contributed to the idea, conception of the case report, and radiological expertise, and helped revise the case report. All authors read and approved the final manuscript.

\section{Availability of data and materials}

All relevant data is included in the manuscript.

\section{Ethics approval and consent to participate}


Jinan Central Hospital Institutional Review Board approved this study and written informed consent was given by the patient. The images are published under agreement of the patient. Conflicts of interest

The authors have no potential conflicts of interest to disclose.

\section{Consent for publication}

All the authors are consent for publication.

\section{References}

1. Li Q, et al. Early Transmission Dynamics in Wuhan, China, of Novel Coronavirus- Infected Pneumonia. N Engl J Med. 2020; 29. [Epub ahead of print]

2. Chen N, et al. Epidemiological and clinical characteristics of 99 cases of 2019 novel coronavirus pneumonia in Wuhan, China: a descriptive study. Lancet. 2020; 395 (10223):507-13.

3. Jin $\mathrm{YH}$, et al. A rapid advice guideline for the diagnosis and treatment of 2019 novel coronavirus (2019-nCoV) infected pneumonia (standard version). Mil Med Res. 2020; 7(1):4.

4. Pan Y, et al. Initial CT findings and temporal changes in patients with the novel coronavirus pneumonia (2019-nCoV): a study of 63 patients in Wuhan, China. Eur Radiol. 2020 Feb 13. [Epub ahead of print]

5. Guan WJ, et al. Clinical Characteristics of Coronavirus Disease 2019 in China. N Engl J Med. 2020 Feb 28. [Epub ahead of print]

6. Wang W, Tang J, Wei F. Updated understanding of the outbreak of 2019 novel coronavirus (2019nCoV) in Wuhan, China. J Med Virol. 2020; 92(4):441-447.

7. Wang C, et al. A novel coronavirus outbreak of global health concern. Lancet. 2020; 395(10223):470473.

8. Fang Y, et al. Sensitivity of Chest CT for COVID-19: Comparison to RT-PCR. Radiology. 2020, 19:200432. [Epub ahead of print]

9. Ai T, et al. Correlation of Chest CT and RT-PCR Testing in Coronavirus Disease 2019 (COVID-19) in China: A Report of 1014 Cases. Radiology. 2020 Feb 26:200642. [Epub ahead of print]

\section{Figures}



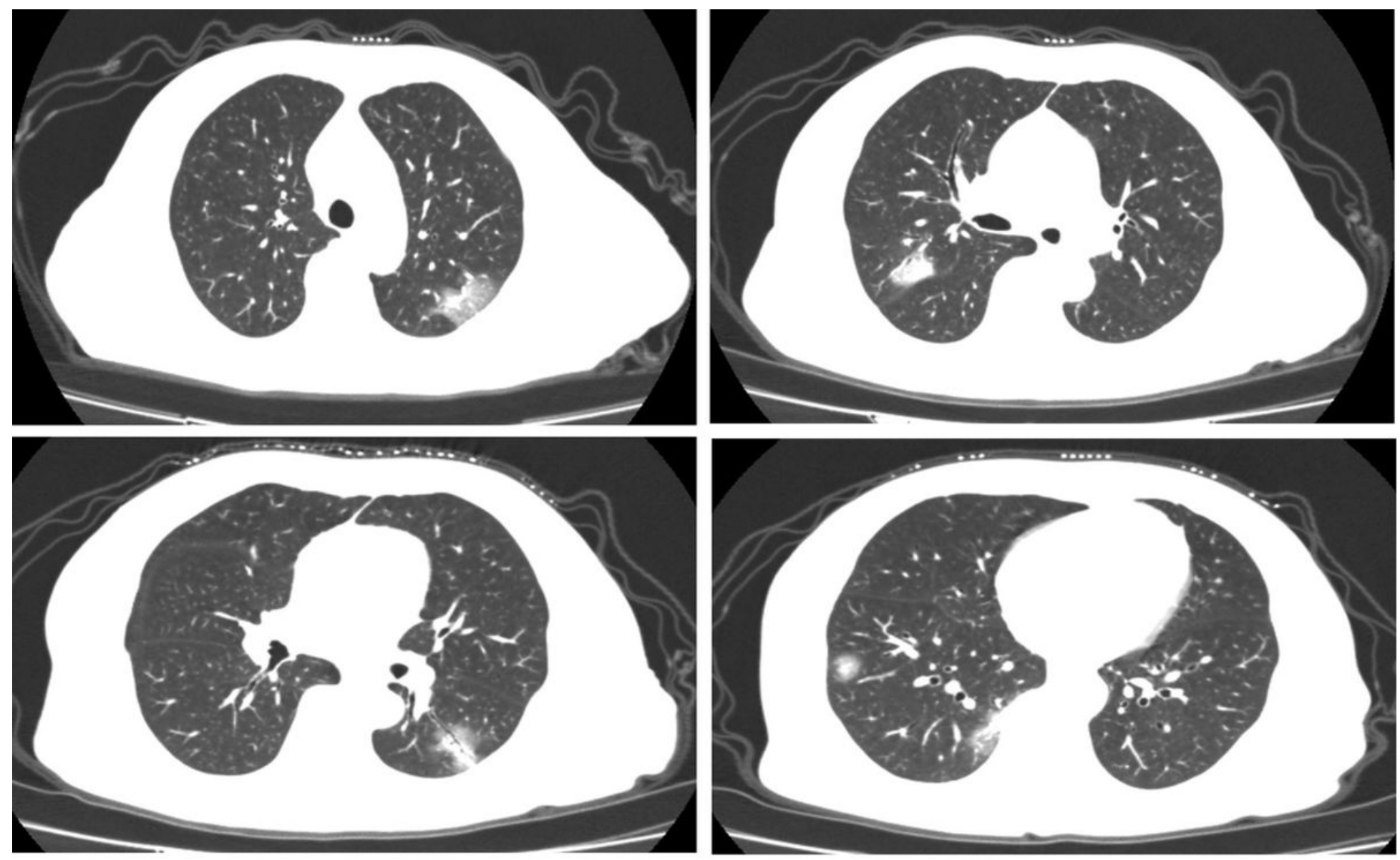

Figure 1

Case 1: CT chest result showed multiple peripheral solid and ground-glass image on bilateral lungs. 

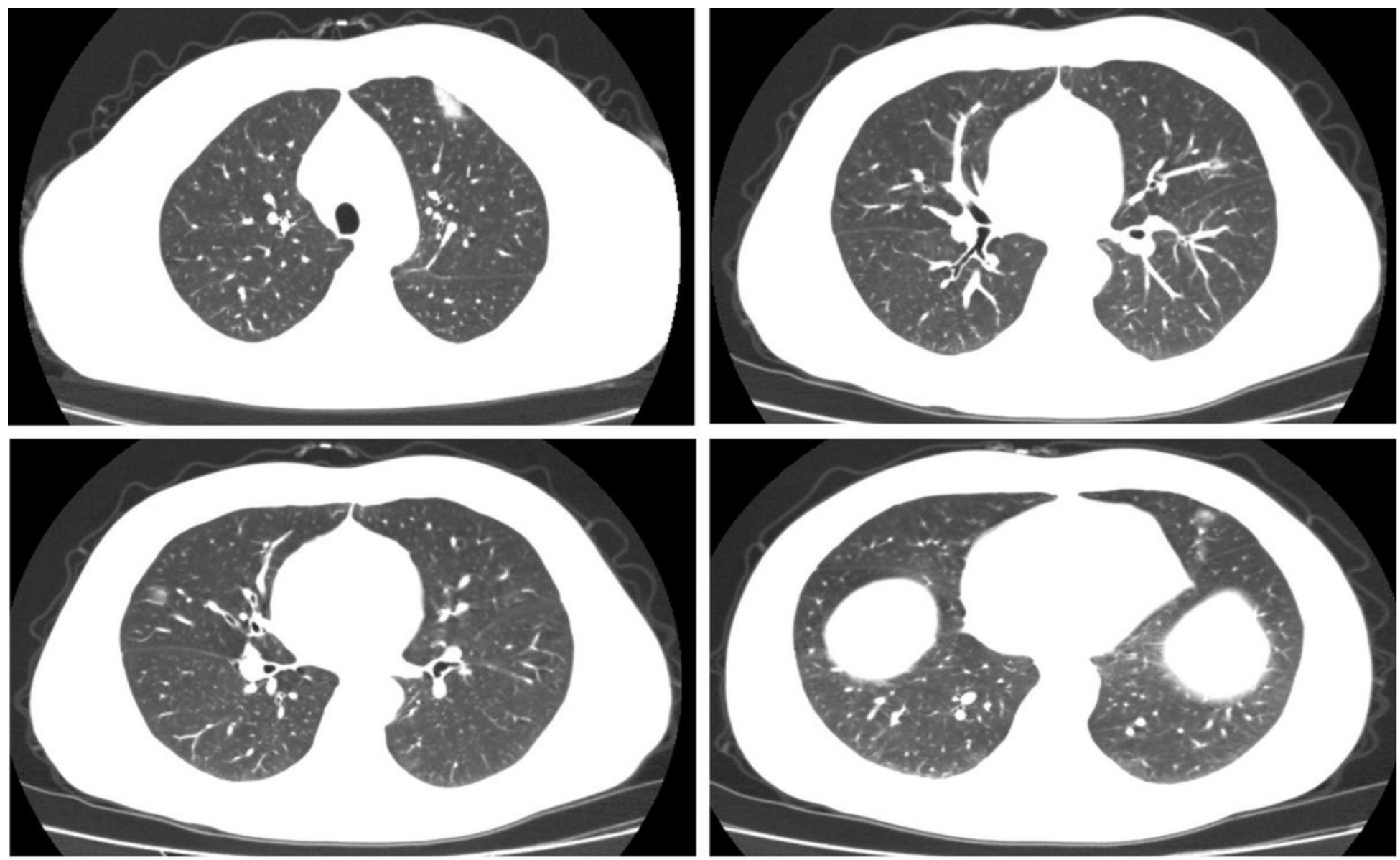

Figure 2

Case 2: CT chest result showed multiple peripheral ground-glass image on bilateral lungs.

New cases

New suspicious cases

18000

15000

12000

9000

6000

3000

0

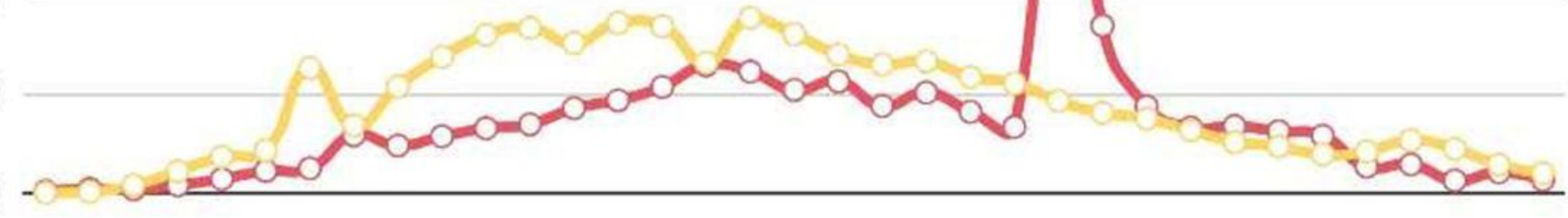

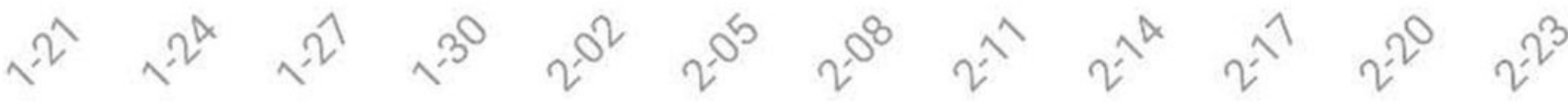




\section{Figure 3}

On February 12, 2020, a total of 15152 new cases in China were confirmed COVID-19 on one day .Among of them, a total of 13332 new cases in Hubei province were clinically confirmed using chest CT without nucleic acid test. 